\title{
Improved Qrs Detector Using Parallel based Hybrid Mamemi Filter
}

\author{
Ramandeep Kaur Bal \\ Guru Nanak Dev University, Amritsar, 143001, India \\ Email: ramandolly83@yahoo.com \\ Anil Kumar \\ Guru Nanak Dev University, Amritsar, 143001, India \\ Email: anil.dcse@gndu.ac.in
}

\begin{abstract}
QRS detection is becoming more popular in detecting the heart beat rate. The improvement is done by using the new filter. The data and control parallelism is used in order to improve the execution time and speed of the parallel based hybrid MAMEMI filter technique This research work focus on providing better performance in heart beat detection algorithm by using parallel hybrid filter.An enhanced algorithm has been proposed to enhance the performance of QRS detection. Different parameters are used for the performance analysis. Accuracy,F_Measure, and Detection_Error_rate are the parameters which are used to evaluate the performance of heart beat algorithm. The results of proposed algorithm are compared with existing heart beat detection algorithm for performance comparison. On the other hand the performance of the proposed method is also improved using parallelism. Parallel proposed method shows better results than Sequential proposed method. The Mean improvement in execution time is 0.80 .
\end{abstract}

Index Terms-Electrocardiogram, QRS detector, hybrid MaMeMi filter, Baseline wander, parallelism.

\section{INTRODUCTION}

\section{A. $Q R S$}

Electronics is playing vital role in the medical field. The wearable Electrocardiogram (ECG) system is an example of it. Electrocardiogram(ECG) is one of the significant tool to detect the heart diseases of a human being. Analysis of the electrocardiogram signals produced by heart results in detection of the abnormalities of the heart. Shape and occurrence time of the ECG signal provide much information about the heart function. The electrocardiogram (ECG) is one of the most significant diagnostic tests in medical field. ECG acts as an crucial device for the safety of the heart patients. Depolarization and Repolarization of the heart results in the formation of the various signal waves .Skin electrodes are of helpful in recording the electrical signals' movement which are shown graphically in Figure 1.

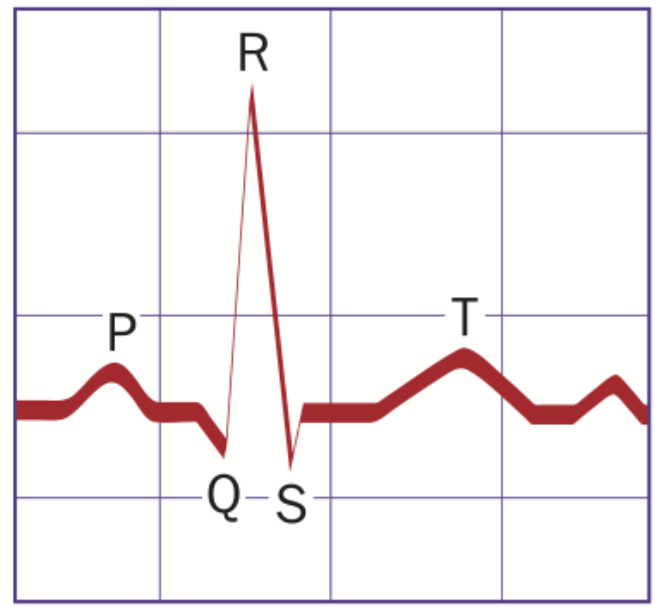

Fig.1. Representation of heart beat signal

\section{B. Data parallelism}

In parallel computing environments data parallelism is a type of parallelization of computing across multiple processors. The main focus of data parallelism is to distribute the data across different parallel processors. When each processor executes the same task on different data sets then it results in the data parallelism. In some situations, a single execution thread controls operations on all pieces of data.

\section{Control parallelism}

In parallel computing environments task parallelism is a form of parallelization of code across multiple computing nodes. The main focus of task parallelism is to distribute tasks across different parallel processor. When each processor executes a different process on the same or different data then it results in the task parallelism.

\section{RELATED WORKS}

Junior et al. (2016) [1] has proposed the new technique to detect the QRS complex. Based upon the Redundant Discrete Wavelet Transform (RDWT) a real time QRS 
complex detector is presented. Both scales and wavelet coefficients for detection are used in this algorithm. Based upon the position of QRS and wavelet coefficients the $\mathrm{P}$ and $\mathrm{T}$ waves can be detected. The QRS detector is evaluated on ECG signals from MIT-BIH database and delivers a detection rate of QRS complex above $99.32 \%$.

Krishna (2015)[2] has proposed the digital differentiators technique to detect the QRS complex in the ECG signal. Electrocardiogram(ECG) is one of the significant tool to detect the heart diseases of a human being. It is an important topic for research from several years to extract QRS part from ECG signal. Digital differentiator is one of the constituent part of the Pan Tompkins method which is widely used to detect the QRS complex in the ECG signal. The working of the Pan Tompkins algorithm is studied in which the digital differentiator block is replaced with the Infinite Impulse Response Digital differentiators. MATLAB is used to carry out all simulations and hence the proposed method shows better results.

Martinez et al.(2015) [3] has proposed the Wavelet packet based algorithm for QRS detection A algorithm based on the wavelet packet for QRS region detection is implemented and designed .Electrocardiographic (ECG) records 100 to 109 of the MIT-BIH Arrhythmia database is used for the Validation .Two nodes from the wavelet packet decomposition in the algorithm are used to reconstruct the ECG signal. The QRS detector is evaluated on ECG signals from MIT-BIH database and delivers a sensitivity and positive predictivity of $99.87 \%$ and $99.85 \%$ respectively.

Liu et al.(2015) [4] has proposed a dual-lead fusion algorithm to detect QRS complex.A new algorithm improves the accuracy and sensitivity of single-lead QRS wave. Simulated with the MIT/BIH database, the algorithm delivers sensitivity and accuracy of $99.87 \%$ and $99.81 \%$ respectively.

Abdelliche (2014) [5] has introduced the complex fractional wavelet to detect QRS complex in ECG signal.The Cole-Cole distribution is used to derive complex fractional wavelet. QRS detection scheme is enhanced with the help of the complex Morlet wavelet function. The proposed method has been evaluated against the records of the MIT-BIH arrhythmia database. The results show the usefulness of the proposed method and its higher accuracy

Nagal and Sharma (2014) [6] has introduced the Kmeans clustering algorithm for QRS detection. Basically an electrocardiogram (ECG) shows the record of the electrical activity of the heart .Many diseases such as conduction abnormalities, cardiac arrhythmias and acute myocardial infarctions can be diagnosed with the help of information provided by the ECG analysis. Heart rate can be determined with the help of ECGs by calculating the time between successive QRS complexes. The proposed method achieves the detection rate of $99.89 \%$.

Nakai et al.(2014)[7] has proposed the QRS detection using template matching. A robust method for the QRS detection is presented in the algorithm .Basically, threshold is used to extract the QRS complex from the
ECG signal. The incidence of misdetection and incorrect detection of QRS-complex can be increased due to the presence of different types of noises. To avoid false detection, a novel template matching algorithm is introduced.

Hernando et al.(2014) [8] has proposed the QRS detection using evolutionary algorithms. As the ECG signals are highly non-stationary and corrupted with noises such as muscular noise and baseline wander, therefore QRS detection remains a challenging task. The evolutionary algorithm is used with the aim to find the set of parameters for QRS detection in very noisy ECG signal. Evolutionary algorithm addresses the parameter optimization. Basically 3 types of real noise contaminates the each ECG signal.A quantitative performance of the detector is evaluated with the help of the cost function which is defined by the mean detection jitter, detection error probability and standard deviation. Mean improvement of $4.6 \%$ is shown by QRS detector with its optimized parameters .Higher improvement is shown by the optimized parameters in the noisy records which is the main advantage of the optimized parameters in noisy environments.

Yang et al. (2012) [9] has proposed new technique. Center differentiation is used as a whitening filer, and the high frequency QRS energy is enhanced by the composite function in this method.A novel threshold selection method is proposed to robustly detect the clinical data. The algorithm is tested against the MITBIH Database and delivers the positive accuracy of $99.67 \%$ and sensitivity of $99.83 \%$.

Ahmad at al.(2012) [10] has proposed combined algorithms for detection of QRS complex. For the preprocessing stage the proposed method makes use of Hillbert transform and Wavelet base. The QRS detector is evaluated on ECG signals from MIT-BIH database and delivers a detection error rate (DER) of 98.7\%.The proposed method shows better results after reducing noise in pre-processing stage.

Zhengzhong et al. (2011) [11] has proposed the QRS complex detection technique. Initially, $50 \mathrm{~Hz}$ power-line interference and baseline wander were rejected. The duration of the QRS complex is measured by the improved Tompkins differential method. Feasibility and reliability of these methods were indicated by the experimental results.

Das et al. (2011) [12] has proposed the algorithm for detection of QRS complexes As significant information is provided by the electrocardiogram (ECG) signal for the detection of the abnormal heart disease, therefore detection of QRS complexes from the ECG signal is the initial step to recognize heart disease. Initially, the proposed method uses the empirical mode decomposition (EMD) for signal enhancement. Afterwards this method uses continuous wavelet transform technique for detection of QRS complex from the enhanced signal. The proposed method is evaluated against the MIT-BIH database and it has been observed that proposed method gives fast computational results and very effective. 


\section{Proposed Method}

The proposed method used in this paper is hybrid filter (i.e. MaMeMi filter and derivative filter). The detailed description of the flow chart is given below in different steps:

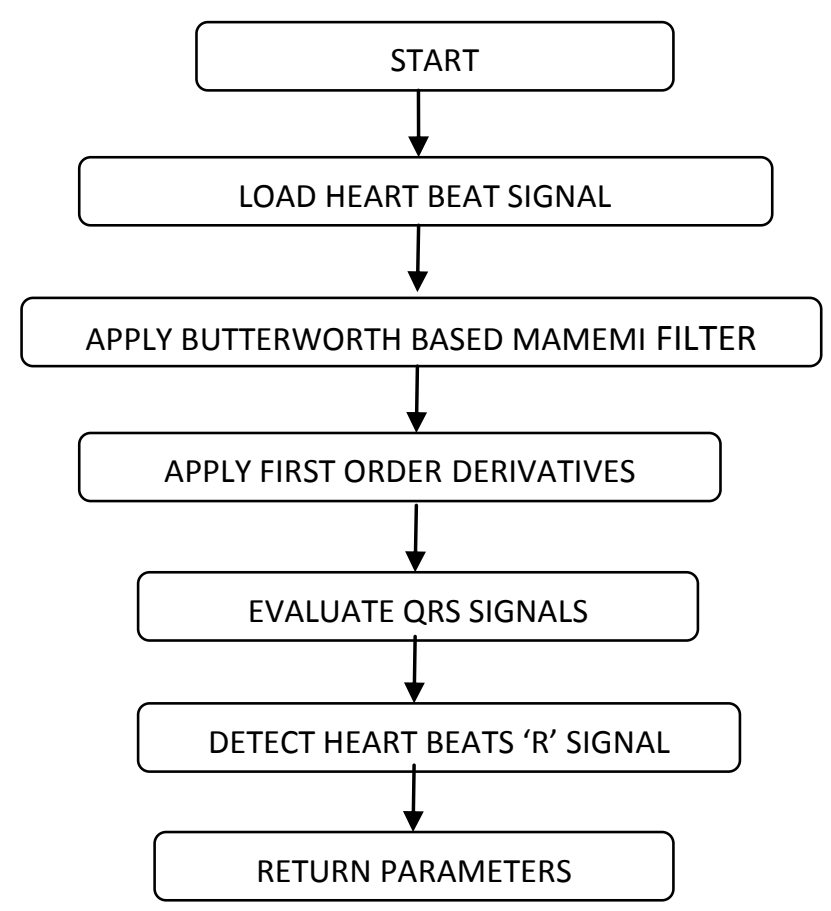

Fig.2. Flowchart of Proposed Methodology

Step1: Start the algorithm.

Step2: Load the heart beat signal which is corrupted with different types of noises. So in order to remove these noises hybrid filter is used.

Step3: Apply Butterworth based MaMeMi filter to remove noises such as baseline wander and high frequency noise.

Step4: Apply First order derivatives which is computationally simple, less time consuming and which shows very accurate results. The equation for derivative is shown in the equation 1.

$$
\mathrm{H}(\mathrm{z})=(1 / 8 \mathrm{~T})\left(-z^{-2}-2 z^{-1}+2 \mathrm{z}+z^{2}\right)
$$

Step5: Evaluate QRS complex in ECG signals. Due to different morphology of QRS complex the R peaks can be positive or negative. $\mathrm{R}$ peaks can only be extreme positive or extreme negative.

Step6: Detect heart beats ' $R$ ' signal. A peak higher than the threshold is considered to be a potential detectable heart beat. Lower peaks are considered as noise.

Step7: Return parameters such as sensitivity and specificity. The results of the proposed method are improved by using hybrid filter which is shown in result section.

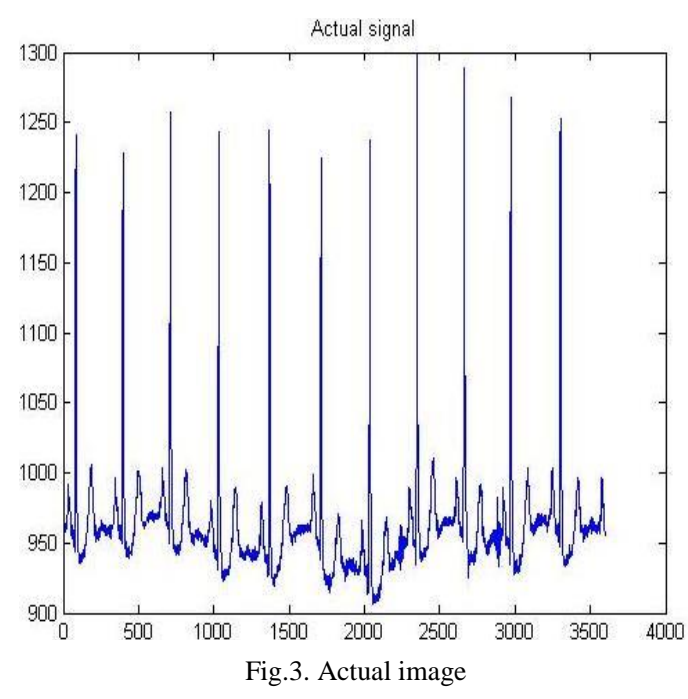

The above image shows the input signal. In this image actual peaks are not cleared as these are attenuated by the baseline wander and high frequency noise.

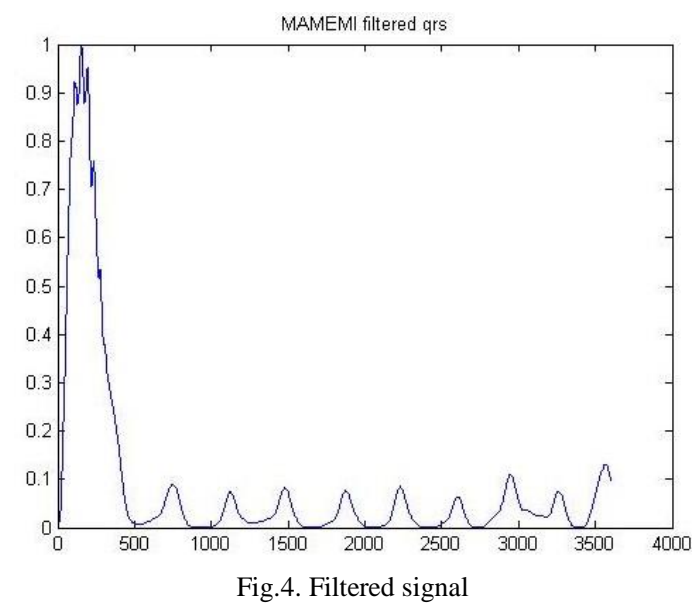

The above image shows the filtered signal after applying the MaMeMi filter in the case of the existing method. MaMeMi filter is basically used to normalize the signal. It is easy to detect the peaks in the normalized signal. It reduces different type of noises present in the signal.

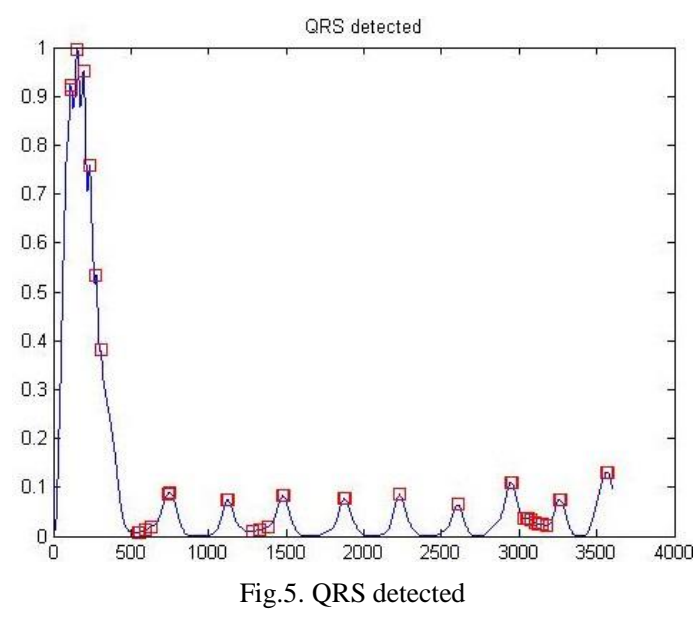


The above image shows the detection of QRS complex. $\mathrm{R}$ peaks are indicated by the red colour.

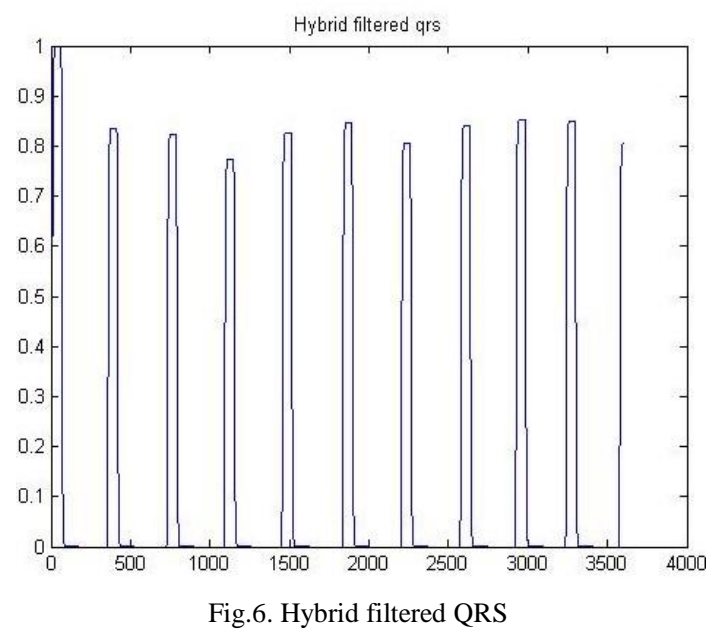

The above image shows the result of the signal after applying the Hybrid filter in the proposed method. Hybrid filter is basically used to normalize the signal. It is easy to detect the peaks in the normalized signal. It reduces the noises more efficiently as compared to existing method.

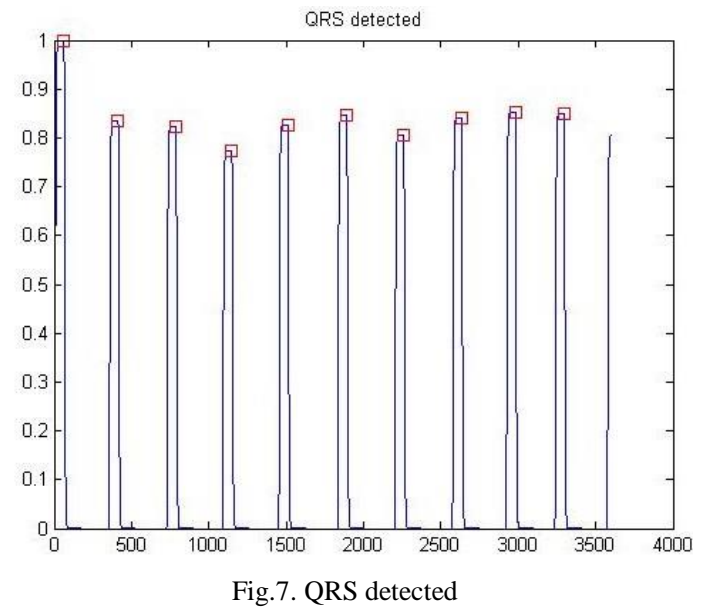

The above image shows the detection of QRS complex.R peaks are indicated by the red color.

\section{RESULTS AND DISCUSSION}

Following are the parameters that are used to improve the performance of the proposed work.

\section{A. Accuracy}

Accuracy identifies the closeness of a calculated value to a standard or known value or Accuracy is defined as, "The ability of a measurement to match the actual value of the quantity being measured". Accuracy is calculated by following formula:

$$
A C C=\frac{T P+T N}{P+N}
$$

where ACC stands for accuracy

TP stands for true positive which is the number of appropriate identifications of $\mathrm{R}$ points within the signal

TN stands for True Negative

$\mathrm{P}$ stands for Positive Instances

$\mathrm{N}$ stands for Negative Instances

Table 1. Comparison table of accuracy

\begin{tabular}{|l|l|l|}
\hline RECORD & $\begin{array}{l}\text { EXISTING } \\
\text { READINGS }\end{array}$ & $\begin{array}{l}\text { PROPOSED } \\
\text { READINGS }\end{array}$ \\
\hline 101 & 0.9493 & 0.9511 \\
\hline 102 & 0.9475 & 0.9502 \\
\hline 104 & 0.9058 & 0.9107 \\
\hline 105 & 0.9018 & 0.9052 \\
\hline 106 & 0.9141 & 0.9327 \\
\hline 107 & 0.7254 & 0.7572 \\
\hline 108 & 0.9510 & 0.9551 \\
\hline 109 & 0.8975 & 0.9052 \\
\hline 111 & 0.9304 & 0.9358 \\
\hline 113 & 0.9128 & 0.9307 \\
\hline 114 & 0.9621 & 0.9670 \\
\hline 116 & 0.8886 & 0.8994 \\
\hline 119 & 0.9197 & 0.9244 \\
\hline 122 & 0.9083 & 0.9120 \\
\hline 124 & 0.9351 & 0.9363 \\
\hline
\end{tabular}

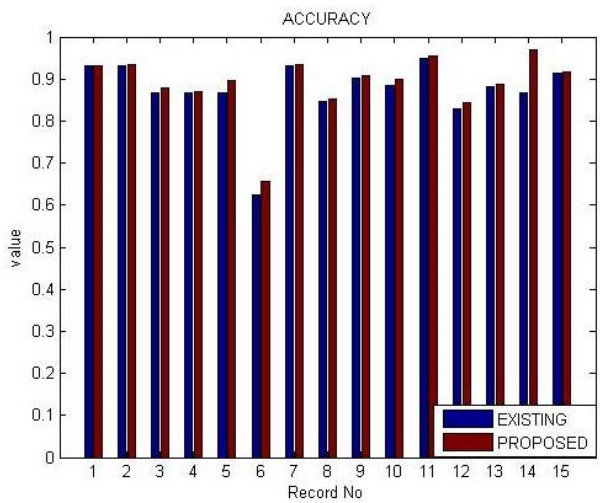

Fig.8. Accuracy Graph

This Fig 8 shows the accuracy graph where the values of proposed method are as high as possible than the existing method.

\section{B. F_Measure}

F-Measure combines and balance recall and precision. It is the compromise between recall and precision. when both are high.

$$
\text { F- Measure }=2 * \frac{P . R}{P+R}
$$

In F-Measure $\mathrm{P}$ is precision and $\mathrm{R}$ is recall values.

The value of F-measure is high. The higher value of Fmeasure shows better is the algorithm. 
Table 2. Comparison table of $\mathrm{f}$ _measure

\begin{tabular}{|l|l|l|}
\hline FILES & $\begin{array}{l}\text { EXISTING } \\
\text { READINGS }\end{array}$ & $\begin{array}{l}\text { PROPOSED } \\
\text { READINGS }\end{array}$ \\
\hline 101 & 0.9306 & 0.9314 \\
\hline 102 & 0.9308 & 0.9339 \\
\hline 104 & 0.8667 & 0.8775 \\
\hline 105 & 0.8664 & 0.8700 \\
\hline 106 & 0.8678 & 0.8950 \\
\hline 107 & 0.6236 & 0.6553 \\
\hline 108 & 0.9308 & 0.9356 \\
\hline 109 & 0.8458 & 0.8519 \\
\hline 111 & 0.9008 & 0.9069 \\
\hline 113 & 0.8833 & 0.8992 \\
\hline 114 & 0.9494 & 0.9544 \\
\hline 116 & 0.8300 & 0.8439 \\
\hline 119 & 0.8808 & 0.8886 \\
\hline 122 & 0.8656 & 0.9700 \\
\hline 124 & 0.9125 & 0.9156 \\
\hline
\end{tabular}

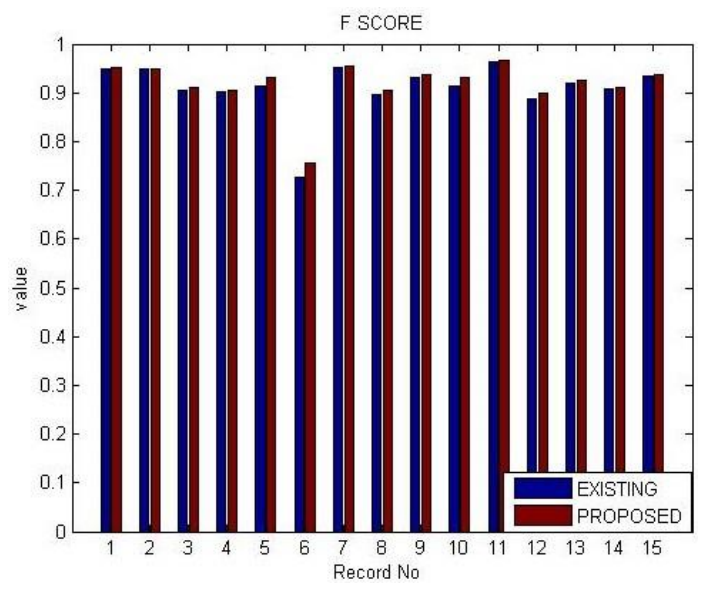

Fig.9. F_Measure Graph

This Fig 9. shows the F_Measure graph where the values of proposed method are as high as possible than the existing method.

\section{Detection_Error_Rate}

The frequency with which errors are presented while detecting QRS complex in ECG signal .Detection Error rate might be measured in terms of incorrect detection of QRS complex from total number of QRS complexes. Error rate is calculated by:

$$
D E R=\frac{F P+F N}{\text { Total } Q R S}
$$

where DER stands for detection error rate

FN stands for false negative that gives number of $\mathrm{R}$ points present in the signal but these are not detected by algorithm
FP stands for false positive that gives number of falsely detected beats.

Total QRS are the number of QRS complex in ECG .

Table 3. Comparison table of detection_error_rate

\begin{tabular}{|c|c|c|}
\hline RECORD & $\begin{array}{c}\text { EXISTING } \\
\text { READINGS }\end{array}$ & $\begin{array}{c}\text { PROPOSED } \\
\text { READINGS }\end{array}$ \\
\hline 101 & 0.0694 & 0.0686 \\
\hline 102 & 0.0692 & 0.0661 \\
\hline 104 & 0.1333 & 0.1225 \\
\hline 105 & 0.1336 & 0.1330 \\
\hline 106 & 0.1322 & 0.1050 \\
\hline 107 & 0.3764 & 0.3447 \\
\hline 108 & 0.0692 & 0.0644 \\
\hline 109 & 0.1542 & 0.1481 \\
\hline 111 & 0.0992 & 0.0931 \\
\hline 113 & 0.1167 & 0.1008 \\
\hline 114 & 0.0506 & 0.0456 \\
\hline 116 & 0.1700 & 0.1561 \\
\hline 119 & 0.1192 & 0.1114 \\
\hline 122 & 0.1344 & 0.1300 \\
\hline 124 & 0.0875 & 0.0844 \\
\hline
\end{tabular}

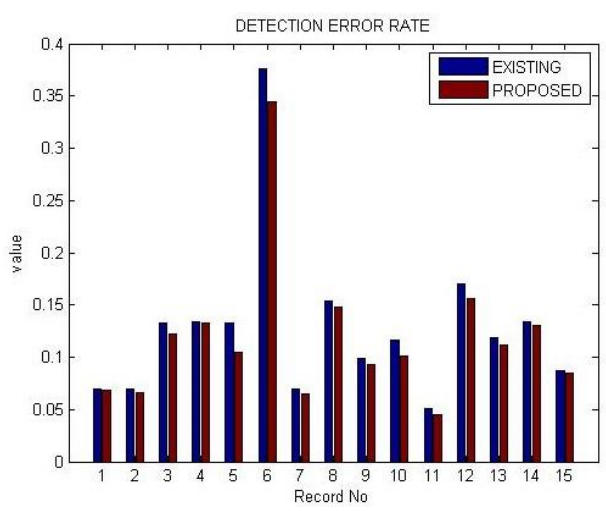

Fig.10. Detection_Error_Rate Graph

This Fig 10. shows the Detection_Error_Rate graph where the values of proposed method are as high as possible than the existing method.

\section{Execution time}

Execution time is defined as the time taken to complete an program after submission to a machine till finish.The execution time is called sequential execution time when the program is submitted to a serial computer and denoted by $\mathrm{T}_{\mathrm{s}}$. The execution time is called parallel execution time when the program is submitted to a parallel computer and denoted by $\mathrm{Tp}$. 
Table 4. Represents the values of Execution Time in Proposed Parallel and Proposed Sequential method..

\begin{tabular}{|l|l|l|}
\hline $\begin{array}{l}\text { RECORD } \\
\text { NO. }\end{array}$ & $\begin{array}{l}\text { PROPOSED } \\
\text { SEQUENTIAL }\end{array}$ & $\begin{array}{l}\text { PROPOSED } \\
\text { PARALLEL }\end{array}$ \\
\hline 101 & 1.9218 & 1.4558 \\
\hline 102 & 1.9943 & 1.3290 \\
\hline 104 & 1.9648 & 1.3694 \\
\hline 105 & 1.9462 & 1.2879 \\
\hline 106 & 1.9273 & 1.2606 \\
\hline 107 & 2.0111 & 1.4472 \\
\hline 108 & 1.9543 & 1.4414 \\
\hline 109 & 2.3652 & 1.3867 \\
\hline 111 & 2.4426 & 1.2802 \\
\hline 112 & 2.4138 & 1.4417 \\
\hline 113 & 2.3578 & 1.3439 \\
\hline 114 & 2.3723 & 1.2170 \\
\hline 115 & 2.3719 & 1.6893 \\
\hline 116 & 2.4149 & 1.6921 \\
\hline 118 & 2.4852 & 1.2433 \\
\hline
\end{tabular}

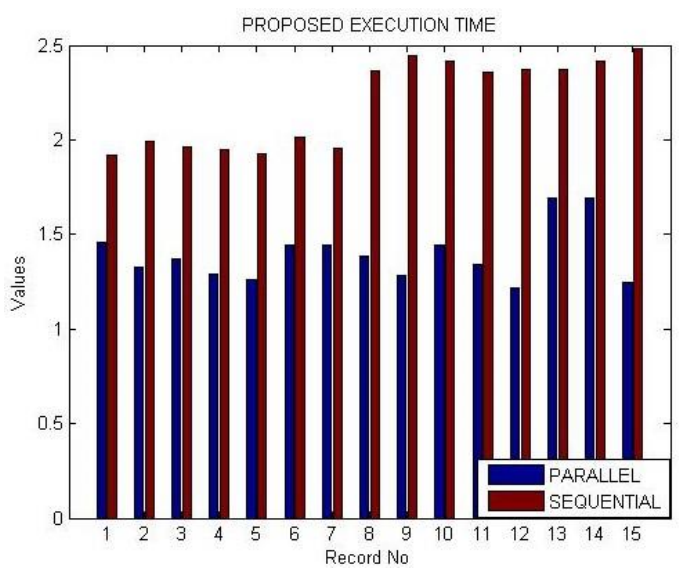

Fig.11. Execution time Graph

This Fig .11. shows the Execution time graph where the values of proposed method are as high as possible than the existing method.

\section{E. $\quad$ Speed up}

Speed up of a parallel algorithm is the ratio of execution time when the computer program is executed sequentially to the execution time when the computer program is executed by more than one processor in parallel. Speed up can be mathematically represented as: $\mathrm{Sp}=\mathrm{Ts} / \mathrm{Tp}$, where $\mathrm{Ts}$ is the sequential execution time, $\mathrm{Tp}$ is the parallel execution time.

For calculating speedup the formula are :

$$
\begin{gathered}
\text { speedup }=\frac{\text { sequential execution time }}{\text { parallel exuction time }} \\
S U=\frac{T_{S}}{T_{P}}
\end{gathered}
$$

\section{F. Efficiency}

It is defined as the ratio of parallel execution time and no of execution units where parallel execution time is a time required by a parallel computers to complete the assigned computer program and it is denoted by $T_{p}$.

For calculating efficiency the formula are

$$
\begin{gathered}
\text { efficiency }=\frac{\text { parallel execution time }}{4} \\
E I=\frac{T_{p}}{4}
\end{gathered}
$$

Table 5. Represents the values of Speedup and efficiency in parallel based proposed method.

\begin{tabular}{|l|l|l|}
\hline $\begin{array}{l}\text { RECORD } \\
\text { NO. }\end{array}$ & EFFICIENCY & SPEEDUP \\
\hline & & \\
\hline 101 & 0.3639 & 1.3200 \\
\hline 102 & 0.3322 & 1.5006 \\
\hline 104 & 0.3423 & 1.4347 \\
\hline 105 & 0.3219 & 1.5111 \\
\hline 106 & 0.3151 & 1.5288 \\
\hline 107 & 0.3618 & 1.3896 \\
\hline 108 & 0.3603 & 1.3558 \\
\hline 109 & 0.3466 & 1.7056 \\
\hline 111 & 0.3200 & 1.9079 \\
\hline 112 & 0.3604 & 1.6742 \\
\hline 113 & 0.3359 & 1.7544 \\
\hline 114 & 0.3042 & 1.9493 \\
\hline 115 & 0.4223 & 1.4040 \\
\hline 116 & 0.4230 & 1.4271 \\
\hline 118 & 0.3108 & 1.9988 \\
\hline & & \\
\hline
\end{tabular}

\section{CONCLUSION}

The data and control parallelism is used in order to improve the execution time and speed of the parallel based hybrid MAMEMI filter technique. In this paper, the comparison between the Sequential proposed method and the parallel proposed method is done for heart beat detection algorithm. Here the Sequential proposed method i.e. (improved QRS detection by using Parallel based hybrid filter) and the Parallel proposed method i.e. (improved QRS detection by hybrid filter) are discussed. Here comparison between the proposed method i.e. (improved QRS detection by using hybrid filter) and the existing method i.e. (MAMEMI Filter) are also discussed.The MATLAB 2011 version is used to carried out the results. The results section proved that the proposed method shows better results than the existing results and proposed method is also improved using parallelism. The mean execution time of the proposed method is improved by 0.80.In future Multiobjective Differential evolution can be used to enhance the results further. 


\section{REFERENCES}

[1] E.Arrais Junior, R. A. de Medeiros Valentim and G. Bezerra Brandao, "Real Time QRS Detection Based on Redundant Discrete Wavelet Transform," in IEEE Latin America Transactions, vol. 14, no. 4, pp. 1662-1668, April 2016.

[2] B. T. Krishna, "QRS detection using digital differentiators," Electrical Engineering and Informatics (ICEEI), 2015 International Conference on, Denpasar, 2015, pp. 356-359.

[3] G. Vega-Martínez, C. Alvarado-Serrano and L. Leija-Salas, "Wavelet packet based algorithm for QRS region detection and R/S wave identification," Electrical Engineering, Computing Science and Automatic Control (CCE), 2015 12th International Conference on, Mexico City, 2015, pp. $1-6$.

[4] Jian Liu, Xinxin Tan, Ci Huang and Xin Ji, "A dual-lead fusion detection algorithm of QRS," Third International Conference on Cyberspace Technology (CCT 2015), Beijing, 2015, pp. 1-6.

[5] F. Abdelliche, A. Charef and S. Ladaci, "Complex fractional and complex Morlet wavelets for QRS complex detection," Fractional Differentiation and Its Applications (ICFDA), 2014 International Conference on, Catania, 2014, pp. 1-5.

[6] Nagal and S. Sharma, "Simultaneous 12-lead QRS detection by K-means clustering algorithm," Recent Advances and Innovations in Engineering (ICRAIE), 2014, Jaipur, 2014, pp. 1-4.

[7] Y. Nakai et al., "Noise tolerant QRS detection using template matching with short-term autocorrelation," 2014 36th Annual International Conference of the IEEE Engineering in Medicine and Biology Society, Chicago, IL, 2014, pp. 34-37.

[8] D. Hernando, R. Bailón, R. Almeida and A. Hernández, "QRS detection optimization in stress test recordings evolutionary algorithms," Computing in Cardiology 2014, Cambridge, MA, 2014, pp. 737-740.

[9] S. R. Yang, S. C. Hsu, S. W. Lu, L. W. Ko and C. T. Lin, "Development of adaptive QRS detection rules based on center differentiation method for clinical application," 2012 IEEE International Symposium on Circuits and Systems, Seoul, 2012, pp. 2071-2074.

[10] I. L. b. Ahmad, M. b. Mohamed and N. A. b. Ab Ghani, "Development of a concept demonstrator for QRS complex detection using combined algorithms," Biomedical Engineering and Sciences (IECBES), 2012 IEEE EMBS Conference on, Langkawi, 2012, pp. 689-693.

[11] G. Zhengzhong, K. Fanxue and Z. Xu, "Accurate and Rapid QRS Detection for Intelligent ECG Monitor," 2011 Third International Conference on Measuring Technology and Mechatronics Automation, Shangshai, 2011, pp. 298301.

[12] M. K. Das, S. Ari and S. Priyadharsini, "On an algorithm for detection of QRS cis omplexes in noisy electrocardiogram signal," 2011 Annual IEEE India Conference, Hyderabad, 2011, pp. 1-5.

\section{Authors' Profiles}

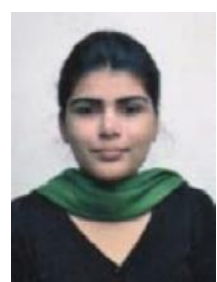

Ramandeep Kaur Bal was born in Amritsar in 1992. She is a M.Tech student of the department computer engineering and technology at Guru Nanak Dev University. She obtained B.Tech degree from Computer science department from Amritsar College of enggineering and technology, Amritsar in 2014. She is currently working on her thesis on the topic QR S detection.

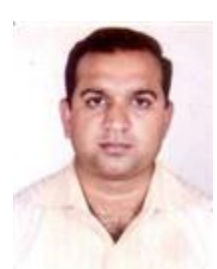

Anil Kumar is an Assistant Professor of the department Computer Engineering and Technology at Guru Nanak Dev University. He received his M.Tech degree from Punjabi University, Patiala in 2004 and B.Tech degree from Guru Nanak Dev University in 2000. He is currently Pursuing Ph.D from Guru Nanak Dev University, Amritsar. His area of interest is in parallel computing.

How to cite this paper: Ramandeep Kaur Bal, Anil Kumar,"Improved Qrs Detector Using Parallel based Hybrid Mamemi Filter", International Journal of Image, Graphics and Signal Processing(IJIGSP), Vol.9, No.3, pp.55-61, 2017.DOI: $10.5815 /$ ijigsp.2017.03.06 